\title{
Radial transport in magnetized non-neutral plasma driven by rotating wave
}

\author{
$\operatorname{AUTHOR}(\mathrm{S})$ :
}

Kiwamoto, Y; Soga, Y; Aoki, J

\section{CITATION:}

Kiwamoto, Y ... [et al]. Radial transport in magnetized non-neutral plasma driven by rotating wave. PHYSICS OF PLASMAS 2005, 12(9): 094501.

\section{ISSUE DATE:}

2005-09

URL:

http://hdl.handle.net/2433/50232

\section{RIGHT:}

Copyright 2005 American Institute of Physics. This article may be downloaded for personal use only. Any other use requires prior permission of the author and the American Institute of Physics. 


\title{
Radial transport in magnetized non-neutral plasma driven by rotating wave
}

\author{
Y. Kiwamoto, ${ }^{\text {a) }}$ Y. Soga, and J. Aoki \\ Graduate School of Human and Environmental Studies, Kyoto University, Yoshida Nihonmatsu-cho, \\ Sakyo-ku, Kyoto 606-8501, Japan
}

(Received 18 May 2005; accepted 22 July 2005; published online 31 August 2005)

\begin{abstract}
Radial transport in non-neutral plasmas driven by a rotating wave field is examined in terms of the drift-kinetic Vlasov equation. It is shown that the radial flux is generated by the $\mathbf{E} \times \mathbf{B}$ drift of resonant particles subject to Landau damping in the axial dynamics. The rate of change in the canonical angular momentum associated with the radial flux is equal to the torque resonantly exerted by the azimuthal component $E_{\theta}$ of the wave. The absorbed wave energy is shared between the axial kinetic energy of the particles and potential energy of the charged particle system. The basic idea of this model may be extended to a scenario that the radial flux is generated by any other dissipative processes that shift the phase relation between the wave and the particle orbits. (C) 2005 American Institute of Physics. [DOI: 10.1063/1.2035427]
\end{abstract}

An appreciable number of observations have been accumulated that indicate the significant contributions rotating electric fields make to the particle transport and to modifications of the density distribution in non-neutral plasmas. ${ }^{1-4}$ While a theoretical model has been proposed for interpretation of the radial transport, more efforts may be necessary toward constructing a clear model for coherent understanding of the processes under the observation. ${ }^{5-7}$ In this Brief Communication we study the transport process in terms of the interaction between the waves and particles starting from the drift-kinetic Vlasov equation and quantitatively examine relations among involved processes.

The present study will reveal that the radial particle flux is generated by the $\mathbf{E} \times \mathbf{B}$ drift of resonant particles that absorb the energy and momentum of the azimuthally traveling waves through Landau damping. The rate of change in the canonical angular momentum associated with the radial flux is shown to be equal to the torque exerted by the azimuthal wave field to the resonant particles. A conservation law holds in the process of the conversion of the wave energy to the potential energy and the kinetic energy of the charged particle system. The authors expect that the present analysis provides a coherent picture about the processes involved in the "rotating wall technique." ${ }^{\text {-3 }}$ It should also be noted that the radial transport as understood here is quite analogous to the physical picture depicted for the radio-frequency flux control of toroidal fusion plasmas. ${ }^{8}$

The configuration we study here consists of a cylindrically symmetric plasma of electrons, with charge $-e$ and mass $m$, immersed in a strong homogeneous magnetic field $\mathbf{B}=B \hat{z}$ and a small-amplitude wave traveling helically with the azimuthal wave number $\ell$ and the axial wave vector $k$. Conversion to the ion plasma case is easily made by replacing the mass and charge. The plasma is infinitely long axially but bounded radially by a co-axial conductor wall placed at $r=w$. We allow arbitrary dependence of the equilibrium density profile $n_{0}(r)$ on the radial coordinate $r$. We assume that

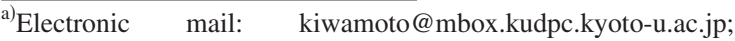

http/:www.plasma.jinkan.kyoto-u.ac.jp
}

URL: the magnetic field is so strong that the transverse motion of the particles is described by the guiding-center approximation:

$$
\frac{\partial f}{\partial t}+\frac{\mathbf{E} \times \mathbf{B}}{B^{2}} \cdot \nabla_{\perp} f+v \frac{\partial f}{\partial z}-\frac{e}{m} E_{\|} \frac{\partial f}{\partial v}=0 .
$$

The equilibrium state is determined by the zeroth order parameters included in the second term, that requests an azimuthal flow $r \omega_{r}(r) \hat{\theta}$ to be driven by the equilibrium electric field $\mathbf{E}_{0}=-\nabla \phi_{0}=E_{0 r} \hat{r}$,

$$
\frac{\mathbf{E}_{0} \times \mathbf{B}}{B^{2}}=-\frac{E_{0 r}}{B} \hat{\theta}=\hat{\theta} \frac{e}{\varepsilon_{0} B} \frac{1}{r} \int_{0}^{r} d r r n_{0}(r)=r \omega_{r}(r) \hat{\theta} .
$$

Here $\hat{\theta}$ stands for the unit vector in the azimuthal direction, and $\omega_{r}(r)$ represents the frequency of the azimuthal rotation that depends on the radial coordinate $r$.

Selecting a Fourier component of $\phi(r) e^{i(\ell \theta+k z-\omega t)}$ from the potential perturbation in Eq. (1), we obtain the perturbation of the velocity distribution function as

$$
\delta f=\frac{\frac{e}{m} k n_{0} \frac{\partial \hat{f}_{0}}{\partial v}-\frac{\ell}{r B} \frac{\partial n_{0}}{\partial r} \hat{f}_{0}}{\omega-\ell \omega_{r}-k v} \phi(r) \equiv-\frac{e}{m} n_{0} \hat{\mathcal{L}}_{f} \phi(r) .
$$

Here $\hat{f}_{0}$ is the zeroth order velocity distribution function normalized to unity on integration over the axial velocity $v$. Introduction of the density perturbation associated with $\delta f$ into the Poisson's equation leads to the differential equation

$$
\nabla \cdot \widetilde{\varepsilon} \cdot \nabla \phi=0 .
$$

Here $\widetilde{\varepsilon}$ is the dielectric tensor given as, ${ }^{9}$

$$
\widetilde{\varepsilon}=\varepsilon_{0}\left(\begin{array}{ccc}
\varepsilon_{\perp} & -i \varepsilon_{X} & 0 \\
i \varepsilon_{X} & \varepsilon_{\perp} & 0 \\
0 & 0 & \varepsilon_{\|}
\end{array}\right),
$$

where $\varepsilon_{0}$ is the dielectric constant in vacuum. The transverse components of the tensor are 


$$
\begin{aligned}
& \varepsilon_{\perp}=1+\frac{\omega_{P}^{2}}{\omega_{C}^{2}}, \\
& \varepsilon_{X}=\frac{\omega_{P}^{2}}{\omega_{C}\left(\omega-\ell \omega_{r}\right)},
\end{aligned}
$$

and the axial component $\varepsilon_{\|}$is

$$
\begin{aligned}
\varepsilon_{\|} & =1-\frac{\omega_{P}^{2}}{k^{2}} \int d v \hat{L}_{f}, \\
& =1-\frac{\omega_{P}^{2}}{k^{2}} \int d v \frac{\frac{\partial \hat{f}_{0}}{\partial v}-\frac{\ell}{\omega_{C} k r n_{0}} \frac{\partial n_{0}}{\partial r} \hat{f}_{0}}{v-\left(\omega-\ell \omega_{r}\right) / k},
\end{aligned}
$$

where $\omega_{P}$ is the local plasma frequency, and $\omega_{C}=e B / m$ is the gyrofrequency.

The explicit form of the Poisson's equation is written as

$$
\frac{1}{r} \frac{\partial}{\partial r}\left(\varepsilon_{\perp} r \frac{\partial \phi}{\partial r}\right)+\frac{\ell}{r} \frac{\partial \varepsilon_{X}}{\partial r} \phi-\frac{\ell^{2}}{r^{2}} \varepsilon_{\perp} \phi-k^{2} \varepsilon_{\|} \phi=0 .
$$

In a non-neutral plasma trapped in a strong magnetic field, we usually see the condition $\omega_{C} \gg \omega_{P}>\omega>\omega_{r}$. If the imaginary part of $\partial \varepsilon_{X} / \partial r$ coming from damping effects in $\omega$ is negligibly small compared to $\varepsilon_{\|}^{\prime \prime}=\operatorname{Im}\left\{\varepsilon_{\|}\right\}$, the imaginary part of $\varepsilon_{\|}$, we obtain

$$
\int_{0}^{w} d r r\left(\varepsilon_{\|}-\varepsilon_{\|}^{*}\right)|\phi|^{2}=0
$$

This relation is derived mathematically by multiplying the complex conjugate of $\phi$ to Eq. (10) and integrating over the radial coordinate from the axis $(r=0)$ to the wall $(r=w)$ after subtracting it from the complex conjugate of its counterpart. By separating the wave frequency $\omega$ into the real part $\omega^{\prime}$ and the imaginary part $\gamma$ and introducing it into Eq. (11), we obtain the growth rate of the wave as

$$
\gamma=-\int_{0}^{w} d r r \varepsilon_{\|}^{\prime \prime}|\phi|^{2} / \int_{0}^{w} d r r \frac{\partial \varepsilon_{\|}^{\prime}}{\partial \omega^{\prime}}|\varphi|^{2} .
$$

Here $\varepsilon_{\|}^{\prime}$ is the real part of $\varepsilon_{\|}$.

Under a strong magnetic field $B$ particles drift radially due to the azimuthal component $E_{\theta}$ of the wave, and the radial drift velocity is given as

$$
\delta v_{r}=\frac{E_{\theta}}{B}=\frac{-i \ell \phi}{r B} .
$$

The radial flux density of the particles is obtained by extracting the time-averaged microflux $\left\langle\delta v_{r} \cdot \delta f\right\rangle$ and integrating it over the axial velocity distribution as

$$
\begin{aligned}
\Gamma & =\int d v\left\langle\delta v_{r} \cdot \delta f\right\rangle=\frac{e n_{0} \ell}{2 m B r}|\phi|^{2} \int d v \operatorname{Im}\left\{\hat{\mathcal{L}}_{f}\right\}, \\
& =-\frac{\varepsilon_{0} \ell k^{2}|\phi|^{2}}{2 e B r} \operatorname{Im}\left\{\varepsilon_{\|}\right\} .
\end{aligned}
$$

The explicit form of the flux density is obtained from Eq. (9) as

$$
\Gamma=\frac{\pi e n_{0} \ell|\phi|^{2}}{2 m B r}\left\{\frac{\partial \hat{f}_{0}}{\partial v}-\frac{\ell}{k r \omega_{c}} \frac{1}{n_{0}} \frac{\partial n_{0}}{\partial r} \hat{f}_{0}\right\}_{v=\left(\omega-\ell \omega_{r}\right) / k} .
$$

This expression is consistent with that obtained by Eggleston and O'Neil. ${ }^{10}$ The first term represents a directional flux that depends on the sense of wave rotation, while the second term indicates the presence of diffusion toward the reduction of particles' concentration. Both terms are proportional to the wave energy density $\propto|\phi|^{2}$ so that the wave-driven diffusion can be stronger than collisional diffusion. When the first term generates the inward flux, the second term tends to limit the sharpening of the density distribution.

The canonical angular momentum consists of the mechanical term and the electromagnetic term as ${ }^{11}$

$$
L_{z}=\sum_{j} m_{j} v_{\theta j} r_{j}+\int_{0}^{w} d r r \oint d \theta r \hat{\theta} \cdot\left(\varepsilon_{0} \mathbf{E} \times \mathbf{B}\right) .
$$

By employing the Poisson's equation for the electric field E, and replacing the summation over discrete particles by spatial integration, $L_{z}$ may be rewritten as

$$
\begin{aligned}
L_{z}= & m \int_{0}^{w} d r 2 \pi r n_{0}(r) r^{2} \omega_{r}(r) \\
& +\frac{m \omega_{C}}{2}\left(N w^{2}-\int_{0}^{w} d r 2 \pi r n_{0}(r) r^{2}\right) .
\end{aligned}
$$

Because the kinetic term is substantially smaller than the electromagnetic term typically by a factor of $\omega_{r} / \omega_{C}<10^{-5}$, the first term is neglected in the following calculation. By using the conservation relation of the number of particles

$$
\frac{\partial n_{0}}{\partial t}+\frac{1}{r} \frac{\partial}{\partial r}(r \Gamma)=0,
$$

the time derivative of the angular momentum is written as follows:

$$
\begin{aligned}
\dot{L}_{z} & =-\frac{m \omega_{C}}{2} \int_{0}^{w} d r 2 \pi r^{3} \frac{\partial n_{0}}{\partial t}, \\
& =\frac{m \omega_{C}}{2} \int_{0}^{w} d r 2 \pi r^{3} \frac{1}{r} \frac{\partial}{\partial r}(r \Gamma) .
\end{aligned}
$$

Substitution of the expression (15) for the flux density leads to

$$
\dot{L}_{z}=\pi \ell k^{2} \varepsilon_{0} \int_{0}^{w} d r r|\phi|^{2} \operatorname{Im}\left\{\varepsilon_{\|}\right\}
$$

Therefore we can estimate the contribution to $\dot{L}_{z}$ from a shell bounded between the radii $r$ and $r+d r$ as

$$
d \dot{L}_{z}=\pi r d r \ell k^{2} \varepsilon_{0}|\phi|^{2} \operatorname{Im}\left\{\varepsilon_{\|}\right\} .
$$

The propagating wave exerts a torque to the plasma contained in the shell ${ }^{12}$ 


$$
d N \hat{z}=2 \pi r d r \vec{r} \times(-e)\left\langle\delta n \mathbf{E}_{\theta}\right\rangle .
$$

Resorting to the same procedure as for Eq. (15), this expression may be rewritten as

$$
d N=\pi r d r \ell k^{2} \varepsilon_{0}|\phi|^{2} \operatorname{Im}\left\{\varepsilon_{\|}\right\} .
$$

Comparison of Eqs. (22) and (24) leads to the relation

$$
d \dot{L}_{z}=d N .
$$

This equation clearly indicates that the canonical angular momentum of the plasma is driven by the torque of the azimuthal electric field of the propagating wave ${ }^{7}$ and that the relevant mechanism is the Landau resonance with the particles streaming in the axial direction.

The modification of the density distribution $n_{0}(r)$ due to the radial flux results in a change in the potential energy $U$ of the particle system. On recalling the potential distribution $\phi_{0}$ of the zeroth order, the increasing rate of $U$ may be written as

$$
\begin{aligned}
\dot{U}= & \int_{0}^{w} d r 2 \pi r\left(-e \phi_{0}\right) \frac{\partial n_{0}}{\partial t}=\int_{0}^{w} d r 2 \pi r\left(-e \phi_{0}\right) \\
& \times\left(-\frac{1}{r} \frac{\partial}{\partial r}(r \Gamma)\right)=-\int_{0}^{w} d r 2 \pi r e \frac{\partial \phi_{0}}{\partial r} \Gamma .
\end{aligned}
$$

Therefore the contribution from the shell bounded within $r$ and $r+d r$ is

$$
\begin{aligned}
d \dot{U} & =-2 \pi r d r e \frac{\partial \phi_{0}}{\partial r} \Gamma \\
& =-\pi r d r \varepsilon_{0} \ell \omega_{r} \omega_{P}^{2}|\phi|^{2} \int d v \operatorname{Im}\left\{\hat{\mathcal{L}}_{f}\right\}, \\
& =\pi r d r \varepsilon_{0} \ell \omega_{r} k^{2}|\phi|^{2} \operatorname{Im}\left\{\varepsilon_{\|}\right\} .
\end{aligned}
$$

Here we have used the relation $\left(\partial \phi_{0} / \partial r\right) / r B=\omega_{r}$ that corresponds to Eq. (2). By combining Eq. (28) with Eq. (24) we obtain the relation between the energy increment and the torque power,

$$
d \dot{U}=\omega_{r} d N
$$

On the other hand the resonant wave-particle interaction in the axial direction should be associated with particle acceleration. The increasing rate of the kinetic energy $d K$ in the shell is evaluated as follows: ${ }^{9}$

$$
\begin{aligned}
d \dot{K}= & 2 \pi r d r \int d v(-e) v\left\langle\delta f E_{z}\right\rangle=\pi r d r \frac{i k e}{2} \int d v v\left(\phi^{*} \delta f\right. \\
& \left.-\phi \delta f^{*}\right), \\
= & -\pi r d r \varepsilon_{0} \omega_{P}^{2} k|\phi|^{2} \int d v v \operatorname{Im}\left\{\hat{\mathcal{L}}_{f}\right\}, \\
= & \pi r d r \varepsilon_{0}\left(\omega-\ell \omega_{r}\right) k^{2}|\phi|^{2} \operatorname{Im}\left\{\varepsilon_{\|}\right\} .
\end{aligned}
$$

By comparing Eqs. (28) and (31) we obtain an interesting relation:

$$
\frac{d K}{d U}=\frac{\omega-\ell \omega_{r}}{\ell \omega_{r}},
$$

and, on integration over $r$, we further obtain

$$
\dot{K}+\dot{U}=-2 \gamma \int_{0}^{w} 2 \pi r d r \varepsilon_{0} \omega^{\prime} \frac{\partial \varepsilon_{\|}^{\prime}}{\partial \omega^{\prime}} \cdot \frac{k^{2}}{4}|\phi|^{2}=-\dot{W} .
$$

Here we have substituted Eq. (12) for $\operatorname{Im}\left\{\varepsilon_{\|}\right\}$. The right-hand side of Eq. (33) stands for the absorption rate of the wave energy due to the Landau damping. ${ }^{9}$ The last relation clearly indicates that the wave energy resonantly absorbed by particles is fully consumed in axial heating and in radial compression and that the branching ratio of the power depends on $r$ as given by Eq. (32).

A few remarks may be in order in comparing the present model with experiments. Rotating waves are launched from an axially localized antenna so that the waves are detected after axial, not temporal, damping. In most cases drastic changes in the density distribution are observed in the time scale much longer than the mean-free-time of Coulomb collision. Therefore one may need to find an appropriate distribution function for $\hat{f}_{0}$ in Eq. (16) so as to include collisional relaxation into quasilinear diffusion process in the phase space $r-v$. In the authors' experiment, a 20-200-fold increase has been observed in the on-axis density when the frequency of the launched wave (in Trivelpiece-Gould mode ${ }^{13,14}$ ) is linearly ramped up over a period much longer than the collision time. The experimentally evaluated radial flux agrees with Eq. (16) within a factor of 3 if we substitute the Maxwellian distribution for $\hat{f}_{0}$ and introduce the global structure of the wave potential $\phi(r)$, as determined from Eq. (10) for the observed density distribution $n_{0}(r)$. When we reverse the sense of rotation, radial expansion of a flattened profile is observed in the density distribution in a manner consistent with Eq. (16). The authors believe that the basic features of the observations are explainable on the basis of the present model. Specific discussion in regard to various experimental conditions will be reported in separate papers.

In closing it may be worth to add a few words about further implication of the present model. ${ }^{15}$ There is a possibility that the basic idea of the model includes other processes than Landau damping. The equations specific to the Landau damping are Eqs. (16) and (31) and (32). The conservation equation (33) can be obtained by combining Eqs. (27) and (30). The essential element in the radial flux $\Gamma$ that is not specific to the Landau damping is $\operatorname{Im}\left\{\hat{\mathcal{L}}_{f}\right\}$ or $\operatorname{Im}\left\{\varepsilon_{\|}\right\}$ originating from the imaginary part of the denominator in Eq. (3). Consequently any dissipative processes that generate $\operatorname{Im}\left\{\hat{\mathcal{L}}_{f}\right\}$ are expected to cause the wave-driven radial flux (16). For quantitative discussion we have to use more general equations that include such processes.

\section{ACKNOWLEDGMENTS}

The author (Y.K.) thanks Professor K. Itoh of NIFS for pointing out the correspondence of the present model with the scenario of the radio-frequency helicity injection in tokamak plasmas. 
This work was supported by the Grant-in-Aid program of JSPS and partly by the collaborative research program of NIFS.

${ }^{1}$ X. -P. Huang, F. Anderegg, C. F. Driscoll, and T. M. O’Neil, Phys. Rev. Lett. 78, 875 (1997).

${ }^{2}$ E. M. Hollmann, F. Anderegg, and C. F. Driscoll, Phys. Plasmas 7, 2776 (2000).

${ }^{3}$ J. R. Danielson and C. M. Surko, Phys. Rev. Lett. 94, 035001 (2005)

${ }^{4}$ Y. Soga, Y. Kiwamoto, A. Sanpei, and J. Aoki, J. Plasma Fusion Res. 6, 715 (2004)

${ }^{5}$ T. M. O'Neil and D. H. E. Dubin, Phys. Plasmas 5, 2163 (1998) (Sec. VI). ${ }^{6}$ D. H. E. Dubin and T. M. O’Neil, Rev. Mod. Phys. 71, 87 (1999) (Sec. III H.2).
${ }^{7}$ R. W. Gould, Nonneutral Plasma Physics III, edited by J. J. Bollinger, R. L. Spencer, and R. C. Davidson, AIP Conf. Proc.498 (AIP, New York, 1999), p. 170.

${ }^{8}$ S. Ioue and K. Itoh, Plasma Physics and Controlled Nuclear Fusion Research 1980 (IAEA, Vienna, 1981), Vol. 2, p. 649.

${ }^{9}$ T. H. Stix, Waves in Plasmas (AIP, New York, 1992), p. 72.

${ }^{10}$ D. L. Eggleston and T. M. O’Neil, Phys. Plasmas 6, 2699 (1999).

${ }^{11}$ T. M. O'Neil, Phys. Fluids 23, 2216 (1980).

${ }^{12} \mathrm{~K}$. Ohta, Introduction to Electrodynamics Vol. II (Maruzen, Tokyo, 2000), p. 325.

${ }^{13}$ A. W. Trivelpiece and R. W. Gould, J. Appl. Phys. 30, 1784 (1959).

${ }^{14}$ R. C. Davidson, Physics of Nonneutral Plasmas (Addison-Wesley, Redwood City, 1990), p. 248.

${ }^{15}$ This part is stimulated by the referee's comment. 